\title{
Prognosis of undiagnosed chest pain: linked electronic health record cohort study
}

\author{
Kelvin P Jordan, ${ }^{1}$ Adam Timmis, ${ }^{2}$ Peter Croft, ${ }^{1}$ Danielle A van der Windt, ${ }^{1}$ Spiros Denaxas, ${ }^{3}$ \\ Arturo González-Izquierdo, ${ }^{3}$ Richard A Hayward, ${ }^{1}$ Pablo Perel, ${ }^{4}$ Harry Hemingway ${ }^{3}$
}

1Arthritis Research UK Primary Care Centre, Research Institute for Primary Care and Health Sciences, Keele University, Keele ST5 5BG, UK

2NIHR Cardiovascular Biomedical Research Unit, Barts Heart Centre, London, UK

${ }^{3}$ Farr Institute of Health Informatics Research London, Institute of Health Informatics, University College London, London, UK

${ }^{4}$ Centre for Global NonCommunicable Diseases, London School of Hygiene and Tropical Medicine, London, UK

Correspondence to: K P Jordan k.p.jordan@keele.ac.uk

Additional material is published online only. To view please visit the journal online.

Cite this as: BMJ 2017;357:j1194 http://dx.doi.org/10.1136/bmj.j1194

Accepted: 21 February 2017

\begin{abstract}
OBJECTIVE

To ascertain long term cardiovascular outcomes in patients whose chest pain remained undiagnosed six months after first presentation.

DESIGN

Cohort study.

SETTING

UK electronic health record database (CALIBER) linking primary care, secondary care, coronary registry, and death registry information.

PARTICIPANTS

172180 adults aged $\geq 18$ from 223 general practices presenting with a first episode of recorded chest pain, classified from medical records as diagnosed (noncoronary condition or angina) or undiagnosed (cause unattributed) at first consultation between 2002 and 2009 and with no previous record of cardiovascular disease.

\section{MAIN OUTCOME MEASURES}

Fatal or non-fatal cardiovascular events over 5.5 years' follow-up. Adjustments were made for age, sex, deprivation, body mass index, smoking status, year of index presentation, and previous records of diabetes or hypertension or previous prescriptions for lipid lowering drugs.

RESULTS
\end{abstract}

At the index presentation, $72.4 \%$ of patients (124688) did not have a cause attributed for their chest pain; 118687 (95.2\%) of these did not receive any type of cardiovascular diagnosis over the next six months. Only a minority of patients in all three groups

\section{WHAT IS ALREADY KNOWN ON THIS TOPIC}

Chest pain in primary care is a common diagnostic challenge with potentially serious consequences if cardiovascular disease is missed

Most patients presenting with new chest pain in primary care do not have a specific diagnosis recorded

Several primary care studies show that people presenting with new chest pain and no diagnosis recorded have a higher risk of a cardiovascular event at one year than those with no chest pain

\section{WHAT THIS STUDY ADDS}

Most people who present with unattributed chest pain have no diagnosis recorded over the next six months and do not undergo diagnostic testing

Patients whose chest pain remains unattributed for six months have higher long term risk of major cardiovascular events than those with an initial non-coronary diagnosis for their chest pain

Because of their numbers, patients with unattributed chest pain after six months have the most myocardial infarctions in the long term and should be targeted for better assessment and cardiovascular disease prevention (non-coronary $2.0 \%$ (769 of 39232$)$; unattributed $11.7 \%$ (14582 of 124688 ); angina $31.5 \%$ (2606 of 8260)) had a recorded cardiac diagnostic investigation in the first six months after presentation. The long term incidence of cardiovascular events was higher in those whose chest pain remained unattributed after six months (5126 of $109628 ; 4.7 \%$ ) compared with patients with an initial diagnosis of non-coronary pain (1073 of $36097 ; 3.0 \%$ ) (adjusted hazard ratios for 0.5-1 year after presentation: $1.95,95 \%$ confidence interval 1.66 to 2.31 ; for $1-3$ years: $1.35,1.23$ to 1.48 ); for $3-5.5$ years: $1.21,1.08$ to 1.37$)$. Owing to the larger number of patients in the unattributed group, there were more excess myocardial infarctions in the long term in this group (214 more than expected based on the rate in the non-coronary group) than in the angina group (132 more than expected). Patients who had cardiac diagnostic investigations in the first six months had a higher long term risk of cardiovascular events, regardless of the initial chest pain label. Incidence of unattributed chest pain and angina decreased between 2002 (124 per 10000 person years and 13 per 10000 person years, respectively) and 2009 (107 per 10000 person years and 5 per 10000 person years, respectively), but the incidence of chest pain attributed to a non-coronary cause remained stable (37-40 per 10000 person years). Risk of cardiovascular events did not change over time.

\section{CONCLUSIONS}

Most patients with first onset chest pain do not have a diagnosis recorded at presentation or in the subsequent six months, including those who undergo cardiac investigations. These patients have an increased risk of cardiovascular events for at least five years. Efforts to better assess and reduce the cardiovascular risk of such patients are warranted.

\section{Introduction}

Each year 1-2\% of adults in the UK attend primary care with chest pain symptoms for the first time..$^{1-3}$ The dominant concern is that these patients may have treatable coronary disease. At the first consultation general practitioners may diagnose myocardial infarction, angina, or a non-coronary cause, such as gastro-oesophageal or musculoskeletal disease or anxiety. Most often the GP will record only the symptom at this stage and not attribute it to any specific cause, ${ }^{124}$ while pursuing investigations in those for whom coronary heart disease is considered a diagnostic possibility.

Cardiovascular disease will be diagnosed in $2-10 \%$ of patients in the unattributed group within 12 months, most within 6-12 weeks of that first presentation. ${ }^{24}$ Risk factors for future cardiovascular events are more 
prevalent in this group than among patients without chest pain. ${ }^{4}$ Patients in the general population who report chest pain and those attending specialist chest pain clinics who are told that they do not have a cardiac cause of their chest pain have a higher future incidence of fatal and non-fatal cardiovascular disease than painfree populations. ${ }^{56}$ Thus, the symptom of chest pain seems to be a marker of cardiovascular risk.

Two important questions remain unanswered. Firstly, do patients who still have unattributed chest pain after an initial period to resolve diagnostic uncertainty have an increased risk of long term fatal and non-fatal cardiovascular events compared with patients diagnosed as having non-coronary chest pain? No study has investigated cardiovascular outcomes in patients with chest pain who remain without a diagnosis of cardiovascular disease, after allowing time for further assessment and short term increases in clinical manifestations of underlying disease. Thus, whether the initial GP diagnosis and investigation provides enough information about disease status to warrant long term monitoring, is unclear.

Secondly, has improved access to diagnostic procedures (such as the introduction of rapid access chest pain clinics) and the observed decrease in the population incidence of coronary heart disease ${ }^{78}$ led to changes in diagnostic labelling and prognosis of chest pain in primary care? This has not been previously investigated.

We sought to tackle these questions by studying patients with no record of previous cardiovascular disease, who consulted and were recorded with first onset of chest pain in UK primary care but were not given an immediate diagnosis of myocardial infarction. We used primary care electronic health records linked to hospital, disease registry, and cause specific mortality records to obtain a more complete and accurate evaluation of major cardiovascular disease endpoints.

\section{Methods}

Setting

The study was set in the CALIBER (cardiovascular disease research using linked bespoke studies and electronic health records) research programme. CALIBER links primary care data from the Clinical Practice Research Datalink (CPRD) to the national registry of acute coronary syndromes (Myocardial Ischaemia National Audit Project, MINAP), inpatient diagnoses and procedures from Hospital Episode Statistics, and cause specific mortality from the Office for National Statistics. ${ }^{9}$ The CPRD is representative of the UK population in terms of sociodemographic characteristics and overall mortality. ${ }^{10-13}$ We have previously demonstrated the validity of CALIBER data for a wide range of cardiovascular risk factors across a variety of incident cardiovascular events. ${ }^{14-17} \mathrm{~A}$ description of the CALIBER approach and phenotyping algorithms combining Read, ICD-10 (international classification of diseases, 10th revision), and drug and procedure codes to define risk factors and endpoints are available at www.caliberresearch.org.

\section{Study population}

The study population was all patients in the database aged 18 or over with a first (incident) coded record of chest pain (denoted as chest pain with cause unattributed, chest pain attributed to non-coronary cause, or angina) in primary or secondary care between 2002 and 2009. We excluded those with a record of angina or cardiovascular disease before their first recorded presentation of chest pain in that period or with less than two years of up-to-standard quality data in the CPRD at the time of their first chest pain event. The first record of chest pain was defined as the index presentation.

\section{Definitions of chest pain at index presentation}

We identified Read and ICD-10 symptom codes (available from www.keele.ac.uk/mrr) through consensus work to define unattributed chest pain (for example, codes for "chest pain not otherwise specified" or "tight chest pain" without clearly specifying a cause of the pain) and non-coronary chest pain (specific attribution to organ systems other than cardiovascular, such as "chest pain oesophagitis"). Read codes record morbidity in UK primary care; ${ }^{18}$ ICD10 codes record morbidity in secondary care. ${ }^{19}$ Angina was defined by Read or ICD10 codes for angina or at least two prescriptions for nitrates.

\section{Alternative explanations for chest pain}

We looked for recorded potential alternative explanations for chest pain in the 24 months before index presentation with a prevalence of at least $1 \%$ in the study population. These were oesophageal reflux, anxiety, depression, chronic obstructive pulmonary disease, chest infection, asthma, osteoarthritis, spinal pain, and cancer. Morbidities were defined using Read and ICD-10 morbidity codes previously developed in CALIBER or through consensus work at Keele University.

\section{Baseline cardiovascular risk factors}

We assessed baseline covariates (age at index presentation, sex, body mass index (BMI), smoking status, neighbourhood deprivation, prescriptions for lipid lowering drugs, and specific comorbidities) for their association with type of recorded chest pain and as potential confounders for the relation between type of chest pain and future cardiovascular disease. BMI and smoking were defined as the closest recorded value before the index date. Deprivation was based on the English index of multiple deprivation 2007 (IMD) ${ }^{20}$ linked to patient postcode, with patients grouped by fifths. The IMD is a weighted aggregate for the local neighbourhood (mean population 1500) of deprivation across seven domains: income; employment; health deprivation and disability; education, skills, and training; barriers to housing and services; living environment; and crime. We identified prescriptions for lipid lowering drugs in the 24 months before the index date. Diabetes and hypertension were included as known risk factors for cardiovascular disease and identified as recorded diagnoses in primary or secondary care in the 24 months before the index presentation. 


\section{Investigations and interventions after index presentation}

We determined the prevalence of cardiac diagnostic investigations (coronary angiography (invasive, computed tomography, magnetic resonance imaging), echocardiography (stress, exercise, and resting), and myocardial perfusion scans) in the first six months after the index date. As a measure of clinical intervention to reduce risk of future cardiovascular disease, we identified prescriptions for lipid lowering, hypertension, and diabetic drugs in the six months after the index presentation up to the first cardiovascular episode or six months, whichever was earlier. Prescriptions for nitrates were not included because they were used to define angina and were regarded as treating a symptom rather than reducing risk.

\section{Outcomes}

We assessed two primary outcomes from index presentation to a maximum follow-up of 5.5 years: incident myocardial infarction (fatal or non-fatal) in all patients and any recorded first fatal or non-fatal cardiovascular event in patients with unattributed or non-coronary chest pain. We identified cardiovascular events in the "diagnostic window" of the first six months after index presentation; the next five years was the period of observation for the long term outcomes, which are the main focus of this paper.

Cardiovascular events were defined as: fatal or non-fatal acute myocardial infarction, angina, coronary heart disease not otherwise specified, heart failure, ventricular arrhythmia, cardiac arrest, ischaemic stroke, haemorrhagic stroke, stroke type not specified, transient ischaemic attack, peripheral arterial disease, abdominal aortic aneurysm, sudden cardiac death, percutaneous coronary intervention, and coronary artery bypass graft surgery. We identified these from primary and secondary care records, MINAP, and the ONS death registry, using previously derived algorithms in CALIBER. ${ }^{9}$ We included all cardiovascular events, rather than just those that may initially present with chest pain, as management of people with definite or possible coronary heart disease includes treatments such as statins or hypertension drugs, which may reduce such events (for example, stroke). This is consistent with recommendations to manage cardiovascular disease as a single family of diseases. ${ }^{21}$

\section{Statistical analysis}

We compared the prevalence of baseline cardiovascular risk factors in the three index groups (unattributed chest pain, non-coronary chest pain, and angina) using multinomial logistic regression, further adjusting for year of index presentation and stratifying by age $(<65$ or $\geq 65$ years). We determined the percentage of patients in each group with a potential alternative explanation for their chest pain.

We compared the incidence of cardiovascular events between groups in two ways. First we derived KaplanMeier curves and crude incidence rates from index presentation to 5.5 years. These, therefore, include events during the initial six month diagnostic period as well as long term outcomes.

Our second, and main, analysis determined the incidence of cardiovascular events in those not diagnosed as having cardiovascular disease in the first six months-that is, the long term prognosis from six months to 5.5 years. Analysis in the unattributed and non-coronary groups was restricted to those with no cardiovascular diagnosis or recorded angina in the first six months and with follow-up data that included at least the first six months. We used Cox proportional hazards regression to determine the risk of long term cardiovascular event by type of chest pain at baseline, adjusting for baseline covariates and year of index presentation. Analysis for the outcome of myocardial infarction included those who presented initially with angina but had no other cardiovascular event in the first six months.

The proportional hazards assumption was assessed using Schoenfeld residuals and deemed adequate for the outcome of myocardial infarction. However, for the outcome of any cardiovascular event, the assumption seemed to be violated, so the follow-up time was split into three periods: six months to one year after initial presentation (all patients), one to three years after initial presentation (in those with no cardiovascular event by one year of follow-up), and three to 5.5 years after the index presentation (in those with no cardiovascular event after three years of follow-up). This seemed to give a better model fit than including a (linear) interaction with time or splitting at other time points. Patients were censored by death, end of follow-up period, or end of records in CALIBER.

We repeated the main analysis in the subgroup of patients who had no record of diagnostic investigations within the first six months.

Finally, we calculated the incidence of new presentations of chest pain for each year from 2002 to 2009. The denominator population for each year was the registered population with at least two previous years of up-to-standard quality data. Annual total incidence rates were directly standardised by applying incidence rates to the age-sex general population structure of England in 2009. However, as this made little difference to the incidence figures, we used crude rates to compare across time.

Robust variance estimators were used in all multivariable analyses, accounting for clustering within general practices. We conducted sensitivity analyses for missing data on the baseline covariates. Analysis was performed using SPSS v.21 and Stata/MP 13.0 for Windows.

\section{Patient involvement}

No patients were involved in setting the research question or the outcome measures, nor were they involved in developing plans for design or implementation of the study. No patients were asked to advise on interpretation or writing up of results. There are no plans to disseminate the results of the research to study participants or the relevant patient community. 


\section{Results}

The denominator population ranged from 1158755 in 2002 to 1417211 in 2009. Of these, 172180 patients from 223 general practices fulfilled the inclusion criteria between 2002 and 2009, with a mean age of 49.0 (SD 17.59). On index presentation, chest pain was recorded as unattributed in 124688 patients $(72.4 \%)$, as non-coronary in $39232(22.8 \%)$, and as angina in 8260 (4.8\%). The index presentation was recorded in primary care for 93.5\% (160911) of patients-39114 in the non-coronary group (99.7\%), 115602 in the unattributed group (92.7\%), and 6195 in the group with angina (75.0\%).

\section{Baseline cardiovascular risk factors}

Fewer than $20 \%$ of the patients with new recorded unattributed or non-coronary chest pain were aged over

\begin{tabular}{|c|c|c|c|c|}
\hline \multirow[b]{2}{*}{ Characteristics } & \multicolumn{3}{|c|}{ Chest pain (No (\%)) } & \multirow[b]{2}{*}{$\begin{array}{l}\text { Total } \\
(n=172180)\end{array}$} \\
\hline & $\begin{array}{l}\text { Non-coronary } \\
(n=39232)\end{array}$ & $\begin{array}{l}\text { Unattributed } \\
(\mathrm{n}=124688)\end{array}$ & $\begin{array}{l}\text { Angina } \\
(\mathrm{n}=8260)\end{array}$ & \\
\hline \multicolumn{5}{|l|}{ Sex: } \\
\hline Female & $22625(57.7)$ & $65652(52.7)$ & $4156(50.3)$ & $92433(53.7)$ \\
\hline Male & $16607(42.3)$ & $59036(47.3)$ & $4104(49.7)$ & $79747(46.3)$ \\
\hline \multicolumn{5}{|l|}{ Age: } \\
\hline $18-44$ & $20159(51.4)$ & $53900(43.2)$ & $563(6.8)$ & $74622(43.3)$ \\
\hline $45-64$ & $12694(32.4)$ & $46498(37.3)$ & $2869(34.7)$ & $62061(36.0)$ \\
\hline $65-74$ & $3588(9.1)$ & $14032(11.3)$ & $2158(26.1)$ & $19778(11.5)$ \\
\hline $75+$ & $2791(7.1)$ & $10258(8.2)$ & $2670(32.3)$ & $15719(9.1)$ \\
\hline \multicolumn{5}{|l|}{ Deprivation fifth: } \\
\hline First (least deprived) & $8328(21.3)$ & $25698(20.7)$ & $1568(19.0)$ & $35594(20.7)$ \\
\hline Second & 7995 (20.5) & $25331(20.4)$ & $1643(20.0)$ & $34969(20.4)$ \\
\hline Third & $7664(19.6)$ & $24825(20.0)$ & $1765(21.4)$ & $34254(20.0)$ \\
\hline Fourth & 7460 (19.1) & $24660(19.8)$ & $1714(20.8)$ & 33834 (19.7) \\
\hline Fifth (most deprived) & $7632(19.5)$ & $23738(19.1)$ & $1545(18.8)$ & $32915(19.2)$ \\
\hline \multicolumn{5}{|l|}{ Risk factors: } \\
\hline Diabetes* & $1278(3.3)$ & $5180(4.2)$ & $1035(12.5)$ & $7493(4.4)$ \\
\hline Hypertension* & $2275(5.8)$ & $11348(9.1)$ & $2526(30.6)$ & $16149(9.4)$ \\
\hline Lipid lowering drug* & $2304(5.9)$ & $10028(8.0)$ & $2123(25.7)$ & $14455(8.4)$ \\
\hline \multicolumn{5}{|l|}{ BMIt: } \\
\hline Normal & $14483(45.9)$ & $42074(41.3)$ & $2114(30.0)$ & $58671(41.7)$ \\
\hline Underweight & $1006(3.2)$ & $2529(2.5)$ & $93(1.3)$ & $3628(2.6)$ \\
\hline Overweight & $10098(32.0)$ & $35261(34.6)$ & $2888(41.0)$ & $48247(34.3)$ \\
\hline Obese & $5962(18.9)$ & $22114(21.7)$ & $1947(27.6)$ & $30023(21.4)$ \\
\hline \multicolumn{5}{|l|}{ Smoking statust: } \\
\hline Non-smoker & $18557(54.1)$ & $59383(53.8)$ & $3868(52.8)$ & $81808(53.8)$ \\
\hline Former smoker & $6199(18.1)$ & $22373(20.3)$ & 2096 (28.6) & $30668(20.2)$ \\
\hline Current smoker & $9540(27.8)$ & $28720(26.0)$ & $1364(18.6)$ & $39624(26.1)$ \\
\hline \multicolumn{5}{|l|}{ Serum total cholesterolt: } \\
\hline$\leq 5$ & $3621(42.0)$ & $14273(39.8)$ & $1721(40.4)$ & $19615(40.2)$ \\
\hline$>5$ & $5007(58.0)$ & $21606(60.2)$ & $2534(59.6)$ & $29147(59.8)$ \\
\hline \multicolumn{5}{|l|}{ Alternative explanations*: } \\
\hline Spinal pain & $8346(21.3)$ & $26164(21.0)$ & $1504(18.2)$ & $36014(20.9)$ \\
\hline COPD/chest infection & $5056(12.9)$ & $16026(12.9)$ & $1264(15.3)$ & $22346(13.0)$ \\
\hline Depression & $3897(9.9)$ & $11780(9.4)$ & $529(6.4)$ & $16206(9.4)$ \\
\hline Gastro-oesophageal reflux & $1964(5.0)$ & $7162(5.7)$ & $445(5.4)$ & $9571(5.6)$ \\
\hline Osteoarthritis & $1527(3.9)$ & $5260(4.2)$ & $694(8.4)$ & $7481(4.3)$ \\
\hline Anxiety & $1643(4.2)$ & $5475(4.4)$ & $243(2.9)$ & $7361(4.3)$ \\
\hline Asthma & $1358(3.5)$ & $4512(3.6)$ & $243(2.9)$ & $6113(3.6)$ \\
\hline Cancer & $942(2.4)$ & $3432(2.8)$ & $422(5.1)$ & $4796(2.8)$ \\
\hline
\end{tabular}

65 (10258 and 6379 patients, respectively), compared with $58.5 \%$ (4828) of patients with new angina (table 1 ). Being male, having previously recorded hypertension, and obesity were associated with unattributed chest pain and with angina rather than with non-coronary chest pain on the index date (see appendix table 1 in supplementary files online). The percentage of patients with potential alternative explanations for their chest pain in their medical records was generally similar between groups (table 1).

Fatal and non-fatal myocardial infarction and any cardiovascular event during follow-up

The median length of follow-up in patients initially recorded with unattributed or non-coronary chest pain $(n=163920)$ was 3.3 years (interquartile range 1.6-5.4). At least six months' follow-up was available for 151317 (92.3\%) patients, and 40010 (24.4\%) patients had the full 5.5 years of follow-up. Kaplan-Meier curves (fig 1) show higher rates of cardiovascular events in the initial six month period than after the six month period and in those with unattributed compared with non-coronary chest pain across the full 5.5 years of follow-up.

Median times to first cardiovascular event were 135 days (interquartile range 22-664) for patients with unattributed chest pain and 665 (248-1165) days for those with non-coronary chest pain. Rates of cardiovascular events were higher in the unattributed group (292 per 10000 person years) than in those in the non-coronary group (107 per 10000 person years) (see appendix table 2). Patients with unattributed chest pain also had a higher rate of myocardial infarction (46 per 10000 person years) than those with non-coronary chest pain (17 per 10000 person years) but a lower rate than for those initially recorded with angina (244 per 10000 person years). Because of the higher number of patients with initially unattributed chest pain, two thirds of myocardial infarctions were in this group (1850 of 2726, 67.9\%). Risk of cardiovascular disease was not statistically significantly different by year of index presentation.

\section{Short term follow-up}

In the first six months after the index date, 2.0\% (769) of those recorded with non-coronary chest pain (39232) and $11.7 \%$ (14582) of those with unattributed chest pain (124668) had a diagnostic investigation (fig 2), compared with $31.5 \%$ (2606) of those recorded with angina (8260). Cardiovascular events were recorded in $4.8 \%$ (6001) of those with unattributed chest pain and 0.7\% (268) of those with initial non-coronary chest pain. Myocardial infarction was recorded in 4.6\% (383) of those with initially recorded angina, $0.7 \%$ (862) of those with unattributed chest pain, and $0.1 \%$ (39) of those with non-coronary pain.

\section{Long term outcomes}

Of the 115014 patients with unattributed chest pain who were followed up for at least six months, 109628 (95.3\%) remained without a cardiovascular diagnosis at six months (fig 2, table 2). Myocardial infarction rate from 0.5 to 5.5 years was highest in the angina group (hazard 


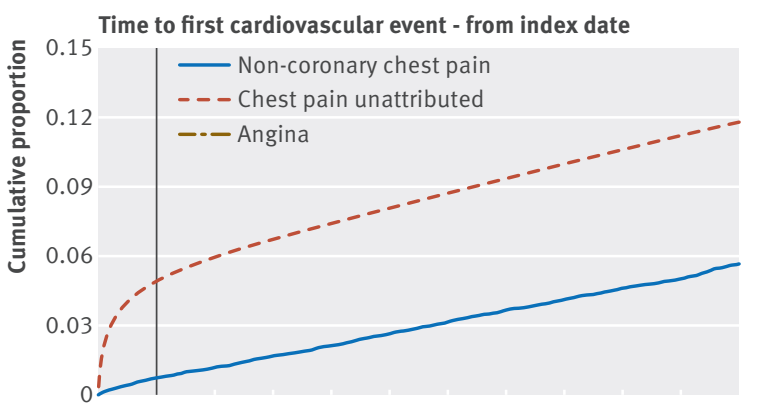

Time to first cardiovascular event in those with no event in first six months

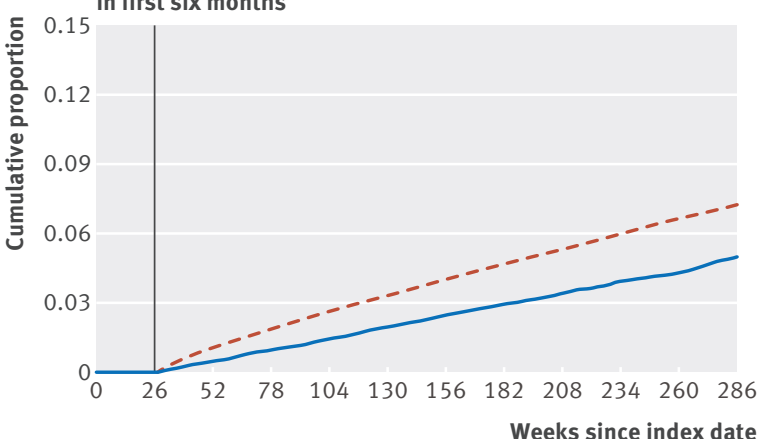

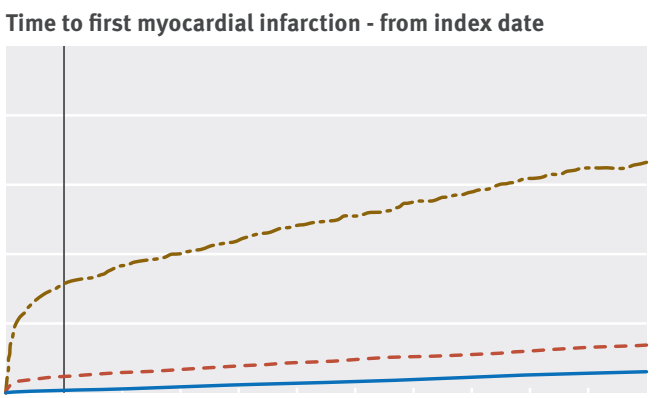

Time to first myocardial infarction in those with no cardiovascular event in first six months

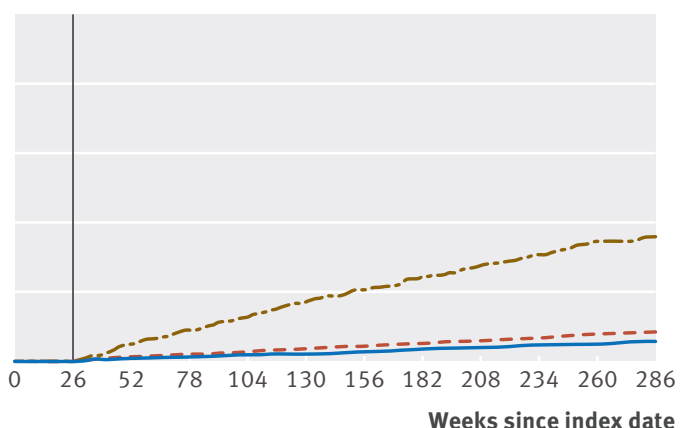

Fig 1 | Kaplan-Meier curves for first cardiovascular event and first myocardial infarction by type of index chest pain

ratio $2.56,95 \%$ confidence interval 2.04 to 3.21 ) and was higher for the unattributed group (1.36, 1.16 to 1.60$)$ compared with the non-coronary group. Owing to the large number of people in the unattributed group, more excess myocardial infarctions occurred from 0.5 to 5.5 years in the unattributed group than in the angina group (214 $v$ 132), when comparing age and sex specific rates of myocardial infarctions to the non-coronary group.

Risk of long term cardiovascular disease was significantly higher over the whole long term follow-up period in patients with initially unattributed chest pain, compared with patients who had a diagnosis of non-coronary pain (0.5-1 year: adjusted hazard ratio $1.95,95 \%$ confidence interval 1.66 to 2.31 ; 3-5.5 years:
1.21, 1.08 to 1.37). Most cardiovascular events took place in the large unattributed group and represented a $43 \%$ excess over expected numbers based on the non-coronary group rate.

The risk of cardiovascular disease in the long term was higher in patients with a diagnostic investigation in the first six months than in those without, regardless of their initial diagnosis (253 per 10000 person years $v 149$ per 10000 for those in the initially unattributed group). Of those with no diagnostic investigation in the first six months, the unattributed group had a higher long term risk of a cardiovascular event (149 per 10000 person years) than the non-coronary group (100 per 10000 (table 2).
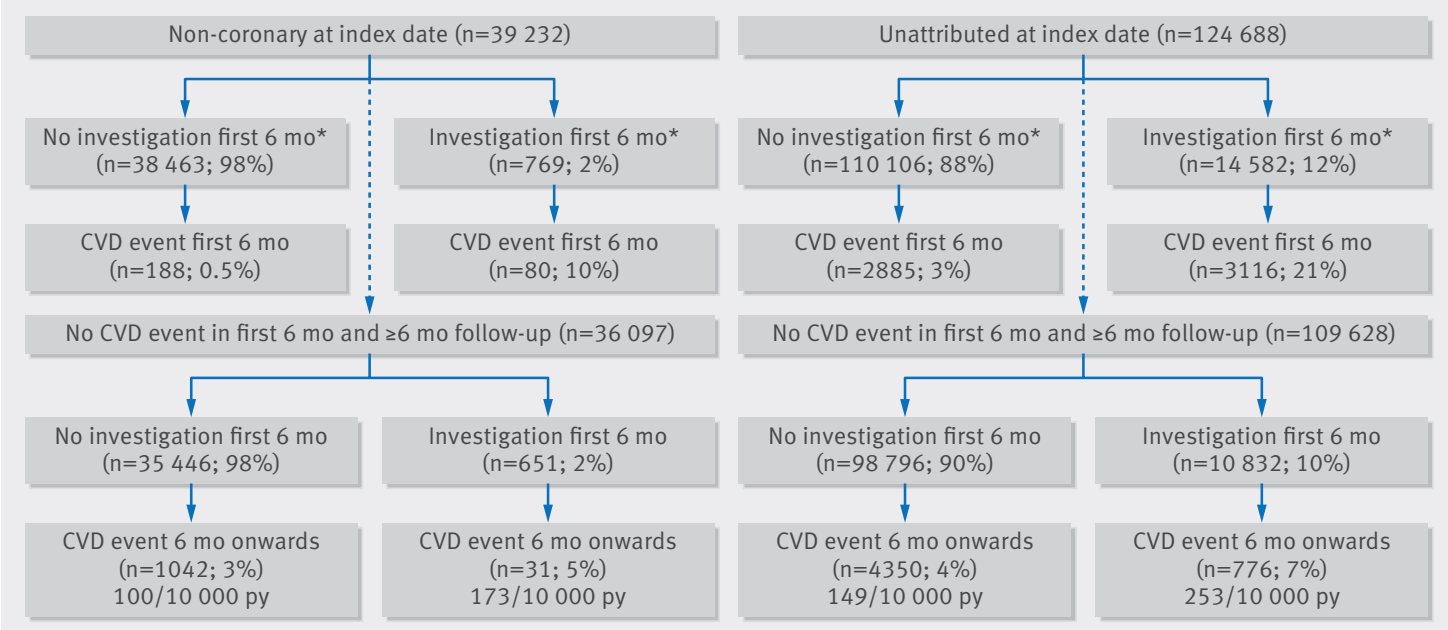

Fig 2 | Cardiovascular events in non-coronary and unattributed groups, stratified by investigations in first six months. $\mathrm{CVD}=$ cardiovascular event; $\mathrm{py}=$ person years. ${ }^{*}$ Before any cardiovascular event 


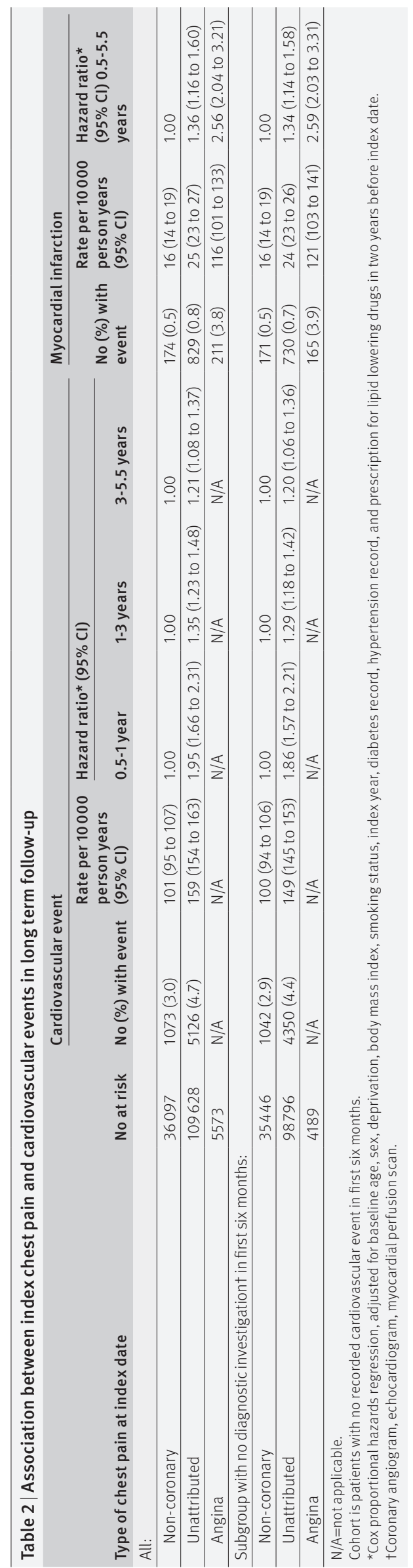

Early intervention after the index consultation

In the six months after the index consultation, and before any cardiovascular event during this period, the proportion of people prescribed lipid lowering, hypertension, or diabetes drugs was $18.3 \%$ (7193 of 39232) and $26.3 \%$ (32812 of 124688 ) in the non-coronary and unattributed groups, respectively. These figures were similar in the subgroup without a recorded cardiovascular event in the first six months (18.1\% (6543 of 36 097) $v 24.9 \%$ (27338 of 109628)) and higher in those without a recorded event who had undergone cardiac investigations (40.1\% (261 of 651) v 42.1\% (4563 of 10832)) (table 3).

\section{Incidence of recorded chest pain}

Incidence of recorded unattributed chest pain decreased from 124 per 10000 person years in 2002 to 107 per 10000 in 2009. Incidence of recorded angina decreased from 13 per 10000 person years to 5 per 10000 over the same period (fig 3). Annual incidence of recorded non-coronary chest pain remained relatively stable (37-40 per 10000). These trends were apparent across both sexes and all age groups (see appendix figure 1). The percentage of all patients with a new record of chest pain who were coded with angina decreased from $7.8 \%$ (1594 of 20478 ) in 2002 to 3.6\% (767 of 21049) in 2009.

\section{Discussion}

Most patients presenting with a new episode of chest pain in primary care did not have their pain attributed to a specific cause by the recording GP, and these patients had a higher rate of subsequent cardiovascular events than those whose chest pain was attributed to a non-coronary cause. Six months later most patients with unattributed chest pain had not undergone diagnostic investigations, had not received drugs that might reduce the risk of cardiovascular disease, and had not experienced a cardiovascular event. For the first time, we have shown that patients in this group have a higher long term incidence of any cardiovascular event and of fatal and non-fatal myocardial infarction than those initially diagnosed as having non-coronary chest pain, for up to five years. Rates of myocardial infarction were highest during this period in those who were initially diagnosed as having angina, but the number of patients with myocardial infarction was highest in the group who had remained undiagnosed at six months because of the total number of people in this group. Prognostic models are needed to identify the patients most at risk, so they can be targeted with specific treatment and lifestyle advice directed at reducing the risk of life threatening cardiovascular events.

We found a decline in incidence of unattributed chest pain and of angina from 2002 to 2009. The trend occurred in all age groups, indicating that it was not caused by changes in coding practices or a cohort effect. These patterns reflect declines in the incidence of cardiovascular disease noted elsewhere and are consistent with unattributed chest pain being a disease marker with important prognostic correlates. ${ }^{78}$ By contrast 


\begin{tabular}{|c|c|c|c|c|}
\hline \multirow[b]{2}{*}{ Interventions } & \multicolumn{2}{|c|}{ Non-coronary at index date } & \multicolumn{2}{|c|}{ Unattributed at index date } \\
\hline & Total & $\begin{array}{l}\text { No (\%) who received } \\
\text { drug in first } 6 \text { months }\end{array}$ & Total & $\begin{array}{l}\text { No (\%) who received } \\
\text { drug in first } 6 \text { months }\end{array}$ \\
\hline \multicolumn{5}{|c|}{ Prescribed lipid lowering, hypertension, or diabetes drugs: } \\
\hline All & 36097 & $6543(18.1)$ & 109628 & $27338(24.9)$ \\
\hline \multicolumn{5}{|l|}{ Prescribed lipid lowering drug: } \\
\hline All & 36097 & $2126(5.9)$ & 109628 & $10067(9.2)$ \\
\hline No investigation in first 6 months & 35446 & $2026(5.7)$ & 98796 & $7962(8.1)$ \\
\hline Investigation in first 6 months & 651 & $100(15.4)$ & 10832 & $2105(19.4)$ \\
\hline
\end{tabular}

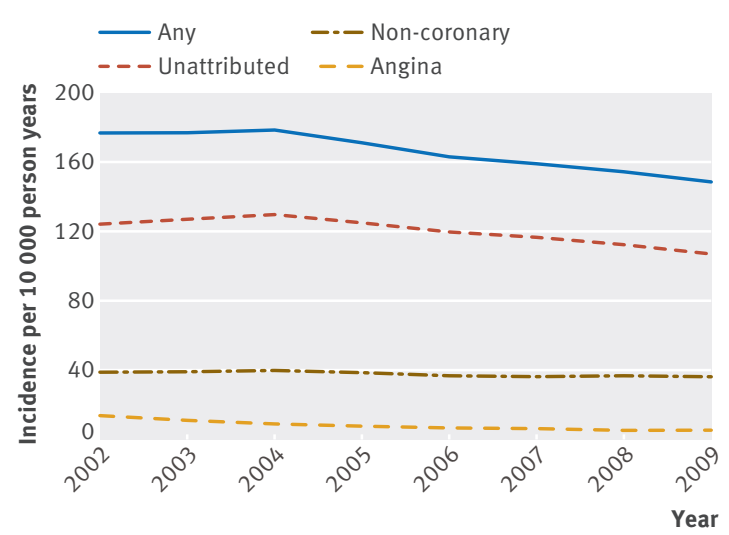

Fig 3 | Trends in incidence of chest pain and angina by year

the incidence of chest pain attributed to a specific non-coronary diagnosis was stable during this period, indicating that GPs are correctly diagnosing patients at low risk of cardiovascular events. The prognostic relation with cardiovascular outcomes did not change across the index years (2002-09), however, suggesting that diagnostic practice and treatment did not change substantially in these years.

\section{Comparison with other studies}

Our findings are presented in the context of previous evidence about the prognosis of patients with chest pain presenting in primary care..$^{1-42223}$ They confirm that angina is a minority diagnosis compared with unattributed chest pain. ${ }^{2424}$ They also confirm that a diagnosis of non-coronary chest pain correctly identifies a low risk group. ${ }^{24}$ Our study is novel in investigating what happens subsequently to patients whose chest pain remains undiagnosed in the first six months after presentation, and we have shown that the initial categorisation is related to future myocardial infarction and to any cardiovascular event for up to five years regardless of whether a diagnostic investigation was performed, reflecting the long term prognostic importance of the initial diagnosis.

\section{Implications}

Our findings were robust to multiple adjustments for baseline differences between the three diagnostic groups. Their importance lies in the large number of patients in whom chest pain was unattributed, accounting for more myocardial infarctions during follow-up than patients with a diagnosis of angina, although incidence rates were substantially lower. Implications for clinical practice might include prevention treatment for all patients with unattributed chest pain, even though most will not go on to experience a cardiovascular event. Another option, favoured by Robson et al, ${ }^{4}$ would be to target patients with unattributed chest pain for more detailed prognostic characterisation to identify those at greatest risk more precisely.

GPs will vary in the proportion and type of patients whose chest pain they attribute to specific causes. We did not assess this variability, although our analyses took into account the clustering of patients in practices. Some GPs may intentionally not record an immediate cardiovascular diagnosis, preferring to wait until diagnosis is confirmed. However, this would not affect our main finding of increased long term risk in those still without a diagnosis after six months. The initial classification of patients as having angina or non-coronary disease identified the groups at highest and lowest risk of future events up to 5.5 years. The decision of the GP to do cardiac investigations identified patients more likely to have or to develop cardiovascular problems, even those given a non-coronary diagnosis for their chest pain. Higher proportions of these patients were taking cardioprotective drugs than those not undergoing investigations.

The "unattributable" label at initial presentation might represent the clinician's diagnostic uncertainty or their decision to delay formal registration of coronary heart disease until investigations have been completed. Investigations are more likely to be recorded in this group than in the non-coronary group and are more likely to identify a subgroup at risk of developing cardiovascular problems. Another message for clinical practice is that patients with chest pain who are investigated but do not have a cardiac disease diagnosed remain at higher risk of future events than those not referred for investigation, confirming results from an earlier study of referrals to chest pain clinic. ${ }^{6}$ This further supports our conclusion that development of prognostic models could help clinical decision making in primary care to identify which patients with chest pain would benefit from early cardiac investigation and which would benefit from preventive treatments. 
Our study shows that initial GP classification of patients with new onset chest pain reflects the likelihood of both underlying current cardiovascular disease and long term risk for future disease. Future research should investigate whether more patients could be placed confidently into the group with initial non-coronary pain, given the low absolute risk of cardiovascular disease in this group. A systematic review of diagnostic indicators of non-cardiovascular chest pain identified several ways in which GP diagnosis of these syndromes might be improved; many patients with unattributed chest pain might be reclassified as definitely having non-coronary chest pain. ${ }^{25}$ Methods to improve diagnosis of coronary heart disease in patients with chest pain would be useful, but clinical algorithms, computerised decision support systems, imaging, and biomarkers have met with variable success in improving the clinical judgment of GPs. ${ }^{426-31}$ Moreover, the evidence shows that patients "ruled out" of a coronary heart disease diagnosis by these methods remain at increased risk of future cardiovascular events. ${ }^{6}$

An alternate aim of research would be to improve estimation of prognosis in patients with unattributed chest pain and to identify which patients are at highest risk of future cardiovascular disease. Interventions and investigations could then be targeted efficiently and effectively. Such research needs to build on existing healthcare information and risk calculators, ${ }^{4}$ new biomarkers, ${ }^{32}$ and development and validation of prognostic models, as has been done for patients with stable angina. ${ }^{33}$ Improving and testing prognostic estimation in this large group of patients in primary care could, given the excess numbers who progress to preventable cardiovascular events, potentially have substantial impact on population health.

\section{Strengths and limitations of this study}

A strength of our study was the large cohort developed within a national (UK) primary care database with linked hospital, myocardial infarction, and death registry data. Estimates of the annual incidence of chest pain over the eight years of the study (15-18 per 1000 person years) were comparable to those from previous analyses of general practice data in the UK. ${ }^{1-4}$ The main limitation, in common with other primary care studies based on routinely collected data, is the lack of specific information on the results of diagnostic testing and the possibility that we have missed testing that was recorded only in secondary care. Although some data were missing for some cardiovascular risk factors, analyses using complete cases and analyses using multiple imputation (not shown) delivered similar findings.

\section{Conclusion}

The large group of patients with undiagnosed chest pain in primary care generally do not undergo diagnostic testing but have an increased risk of fatal and non-fatal cardiovascular events for at least five years. More needs to be done to improve the assessment of chest pain in this group and reduce the cardiovascular risk of such patients.
Contributors: KPJ, AT, PC, DAvW, PP, and HH designed the study. KPJ wrote the analysis plan and performed the analysis. SD and AG-I prepared the data. KPJ, SD, and RAH defined code lists for exposure, covariates, and outcomes. All authors interpreted the findings. KPJ and PC drafted the paper. All authors contributed to revision of the paper and have approved the final version. KPJ is guarantor. The authors had full access to all of the data in the study and can take responsibility for the integrity of the data and the accuracy of the data analysis.

Funding: This study was supported by PROGRESS; Medical Research Council Prognosis Research Strategy (PROGRESS) Partnership (G0902393/99558). The funders had no role in the design of the study or in the collection, analysis, and interpretation of data. Farr Institute of Health Informatics Research was funded by awards from The Medical Research Council [K006584/1], in partnership with Arthritis Research UK, the British Heart Foundation, Cancer Research UK, the Economic and Social Research Council, the Engineering and Physical Sciences Research Council, the National Institute of Health Research, the National Institute for Social Care and Health Research (Welsh Assembly Government), the Chief Scientist Office (Scottish Government Health Directorates), and the Wellcome Trust.

Competing interests: All authors have completed the ICMJE uniform disclosure form at www.icmje.org/coi_disclosure.pdf and declare that the study was supported by a grant from the Medical Research Council, no financial relationships with any organisations that might have an interest in the submitted work in the previous three years; no other relationships or activities that could appear to have influenced the submitted work. This study is based in part on data from the Clinical Practice Research Datalink database obtained under licence from the UK Medicines and Healthcare products Regulatory Agency. This study includes data collected on behalf of the Myocardial Ischaemia National Audit Project (MINAP) under the auspices of the National Institute for Cardiovascular Outcomes Research (NICOR). However, the interpretation and conclusions contained in this report are those of the authors alone.

Ethical approval: This study was approved by the MINAP Academic Group (14-CBR-01) and by the CPRD Independent Scientific Advisory Committee (13_169)

Data sharing: No additional data are available

Transparency: The lead author (KPJ) affirms that this manuscript is an honest, accurate, and transparent account of the study being reported; that no important aspects of the study have been omitted; and that any discrepancies from the study as planned have been explained.

This is an Open Access article distributed in accordance with the terms of the Creative Commons Attribution (CC BY 3.0) license, which permits others to distribute, remix, adapt and build upon this work, for commercial use, provided the original work is properly cited. See: http://creativecommons.org/licenses/by/3.0/

Ruigómez A, Rodríguez LAG, Wallander M-A, Johansson S Jones R. Chest pain in general practice: incidence, comorbidity and mortality. Fam Pract 2006;23:167-74. doi:10.1093/fampra/ cmi124

2 Walters K, Rait G, Hardoon S, Kalaitzaki E, Petersen I, Nazareth I. Socio-demographic variation in chest pain incidence and subsequent coronary heart disease in primary care in the United Kingdom. Eur J Prev Cardiol 2014:21:566-75. doi:10.1177/2047487312449415.

3 Ruigómez A, Massó-González EL, Johansson S, Wallander MA, García-Rodríguez LA. Chest pain without established ischaemic heart disease in primary care patients: associated comorbidities and mortality. BrJ Gen Pract 2009;59:e78-86. doi:10.3399/ bjgp09X407054.

4 Robson J, Ayerbe L, Mathur R, Addo J, Wragg A. Clinical value of chest pain presentation and prodromes on the assessment of cardiovascular disease: a cohort study. BMJ Open 2015;5:e007251. doi:10.1136/bmjopen-2014-007251.

5 Croft PR, Thomas E. Chest pain and subsequent consultation for coronary heart disease: a prospective cohort study. Br J Gen Pract 2007;57:40-4.

6 Sekhri N, Feder GS, Junghans C, Hemingway H, Timmis AD. How effective are rapid access chest pain clinics? Prognosis of incident angina and non-cardiac chest pain in 8762 consecutive patients. Heart 2007;93:458-63. doi:10.1136/hrt.2006.090894.

7 Davies AR, Smeeth L, Grundy EM. Contribution of changes in incidence and mortality to trends in the prevalence of coronary heart disease in the UK: 1996 2005. Eur Heart J 2007;28:2142-7. doi:10.1093/eurheartj/ehm272.

8 Smolina K, Wright FL, Rayner M, Goldacre MJ. Determinants of the decline in mortality from acute myocardial infarction in England between 2002 and 2010: linked national database study. BM 2012;344:d8059. doi:10.1136/bmj.d8059. 
9 Denaxas SC, George J, Herrett E, et al. Data resource profile: cardiovascular disease research using linked bespoke studies and electronic health records (CALIBER). Int I Epidemiol 2012:41:1625-38. doi:10.1093/ije/dys188.

10 Gallagher A, Puri S, van Staa T. Linkage of the general practice research database (GPRD) with other data sources. Pharmacoepidemiol Drug Saf 2011;20:S230-1.

11 Mathur R, Bhaskaran K, Chaturvedi N, et al. Completeness and usability of ethnicity data in UK-based primary care and hospital databases. Public Health (Oxf) 2014:36:684-92. doi:10.1093/pubmed/fdt116.

12 Herrett E, Gallagher AM, Bhaskaran K, et al. Data resource profile: Clinical practice research datalink (CPRD). Int J Epidemiol 2015:44:827-36. doi:10.1093/ije/dyv098.

13 Herrett E, Shah AD, Boggon R, et al. Completeness and diagnostic validity of recording acute myocardial infarction events in primary care, hospital care, disease registry, and national mortality records: cohort study. BM/ 2013;346:f2350. doi:10.1136/bmj.f2350.

14 George J, Rapsomaniki E, Pujades-Rodriguez M, et al. How does cardiovascular disease first present in women and men? Incidence of 12 cardiovascular diseases in a contemporary cohort of 1937360 people. Circulation 2015;132:1320-8. doi:10.1161/CIRCULATIONAHA.114.013797.

15 Rapsomaniki E, Timmis A, George J, et al. Blood pressure and incidence of twelve cardiovascular diseases: lifetime risks, healthy life-years lost, and age-specific associations in 1.25 million people. Lancet 2014;383:1899-911. doi:10.1016/S0140-6736(14)60685-1.

16 Shah AD, Langenberg C, Rapsomaniki E, et al. Type 2 diabetes and incidence of cardiovascular diseases: a cohort study in 1.9 million people. Lancet Diabetes Endocrinol 2015;3:105-13. doi:10.1016/ S2213-8587(14)70219-0.

17 Pujades-Rodriguez M, George J, Shah AD, et al. Heterogeneous associations between smoking and a wide range of initia presentations of cardiovascular disease in 1937360 people in England: lifetime risks and implications for risk prediction. Int J Epidemiol 2015:44:129-41. doi:10.1093/ije/dyu218.

18 Health and Social Care Information Centre. Read codes. 2014 http://systems.hscic.gov.uk/data/uktc/readcodes.

19 World Health Organization. Classification of diseases. http://apps. who.int/classifications/icd/en/index.html.

20 Department for Communities and Local Government. The English indices of deprivation 2007: summary. 2007. https://www.gov.uk/ government/collections/english-indices-of-deprivation.

21 Department of Health. Cardiovascular disease outcomes strategy: improving outcomes for people with or at risk of cardiovascular disease. 2013. https://www.gov.uk/government/publications/ improving-cardiovascular-disease-outcomes-strategy

22 Hemingway H, Shipley M, Britton A, Page M, Macfarlane P, Marmot M. Prognosis of angina with and without a diagnosis: 11 year follow up in the Whitehall II prospective cohort study. BMJ 2003;327:895. doi:10.1136/bmj.327.7420.895.
23 Herrett E, George J, Denaxas S, et al. Type and timing of heralding in ST-elevation and non-ST-elevation myocardial infarction: an analysis of prospectively collected electronic healthcare records linked to the national registry of acute coronary syndromes. Eur Heart J Acute Cardiovasc Care 2013;2:235-45. doi:10.1177/2048872613487495.

24 Svavarsdóttir AE, Jónasson MR, Gudmundsson GH, Fjeldsted K. Chest pain in family practice. Diagnosis and long-term outcome in a community setting. Can Fam Physician 1996;42:1122-8.

25 Wertli MM, Ruchti KB, Steurer J, Held U. Diagnostic indicators of non-cardiovascular chest pain: a systematic review and metaanalysis. BMC Med 2013;11:239. doi:10.1186/1741-7015-11-239.

26 Haasenritter J, Donner-Banzhoff N, Bösner S. Chest pain for coronary heart disease in general practice: clinical judgement and a clinical decision rule. BrJ Gen Pract 2015;65:e748-53. doi:10.3399/ bjgp15X687385

27 Johnson R, Evans M, Cramer H, et al. Feasibility and impact of a computerised clinical decision support system on investigation and initial management of new onset chest pain: a mixed methods study. BMC Med Inform Decis Mak 2015;15:71. doi:10.1186/ s12911-015-0189-8.

28 Poldervaart JM, Röttger E, Dekker MS, et al. No added value of novel biomarkers in the diagnostic assessment of patients suspected of acute coronary syndrome. PLoS One 2015;10:e0132000. doi:10.1371/ journal.pone. 0132000

29 Westwood M, van Asselt T, Ramaekers B, et al. High-sensitivity troponin assays for the early rule-out or diagnosis of acute myocardial infarction in people with acute chest pain: a systematic review and cost-effectiveness analysis. Health Technol Assess 2015;19:1-234. doi:10.3310/hta19960.

30 Nilsson S, Andersson A, Janzon M, Karlsson JE, Levin LÅ. Cost consequences of point-of-care troponin T testing in a Swedish primary health care setting. Scand J Prim Health Care 2014;32:241-7. doi:10.3109/02813432.2014.984901.

31 Muhlestein JB, Lappé DL, Lima JA, et al. Effect of screening for coronary artery disease using CT angiography on mortality and cardiac events in high-risk patients with diabetes: the FACTOR-64 randomized clinical trial. JAMA 2014;312:2234-43. doi:10.1001/ jama.2014.15825.

32 Cullen L, Greenslade JH, Carlton EW, et al. Sex-specific versus overall cut points for a high sensitivity troponin I assay in predicting 1-year outcomes in emergency patients presenting with chest pain. Heart 2016;102:120-6. doi:10.1136/heartjnl-2015-308506.

33 Rapsomaniki E, Shah A, Perel P, et al. Prognostic models for stable coronary artery disease based on electronic health record cohort of 102 023 patients. Eur Heart/ 2014:35:844-52. doi:10.1093/eurhearti/eht533.

Supplementary information: Supplementary tables and figure 